\title{
Abstract: Learning to Avoid Poor Images Towards Task-Aware C-Arm Cone-Beam CT Trajectories
}

\author{
Jan-Nico Zaech ${ }^{1,2,3}$, Cong Gao ${ }^{1}$, Bastian Bier ${ }^{1,2}$, Russell Taylor ${ }^{1}$, \\ Andreas Maier ${ }^{2}$, Nassir Navab ${ }^{1}$, Mathias Unberath ${ }^{1}$ \\ ${ }^{1}$ Laboratory for Computational Sensing and Robotics, Johns Hopkins University \\ ${ }^{2}$ Pattern Recognition Lab, Friedrich-Alexander-Universität Erlangen-Nürnberg \\ ${ }^{3}$ Computer Vision Laboratory, Eidgenössische Technische Hochschule Zürich \\ unberath@jhu.edu
}

Metal artifacts in computed tomography (CT) arise from a mismatch between physics of image formation and idealized assumptions during tomographic reconstruction. These artifacts are particularly strong around metal implants, inhibiting widespread adoption of 3D cone-beam CT (CBCT) despite clear opportunity for intra-operative verification of implant positioning, e.g. in spinal fusion surgery. On synthetic and real data, we demonstrate that much of the artifact can be avoided by acquiring better data for reconstruction in a task-aware and patient-specific manner, and describe the first step towards the envisioned task-aware CBCT protocol. The traditional short-scan CBCT trajectory is planar, with little room for scene-specific adjustment. We extend this trajectory by autonomously adjusting out-of-plane angulation. This enables C-arm source trajectories that are scene-specific in that they avoid acquiring "poor images", characterized by beam hardening, photon starvation, and noise. The recommendation of ideal out-of-plane angulation is performed on-the-fly using a deep convolutional neural network that regresses a detectability-rank derived from imaging physics. This work was first presented at MICCAI 2019 [1].

\section{References}

1. Zaech JN, Gao C, Bier B, et al. Learning to avoid poor images: towards taskaware c-arm cone-beam CT trajectories. In: Proc - MICCAI 2019. Cham: Springer International Publishing; 2019. p. 11-19. 